\title{
LXII. Hamilton's principle and the five aberrations of von Seidel
}

\section{Lord Rayleigh O.M. Pres.R.S.}

To cite this article: Lord Rayleigh O.M. Pres.R.S. (1908) LXII. Hamilton's principle and the five aberrations of von Seidel , Philosophical Magazine Series 6, 15:90, 677-687, DOI: $10.1080 / 14786440809463809$

To link to this article: http://dx.doi.org/10.1080/14786440809463809

曲 Published online: 16 Apr 2009.

Submit your article to this journal $[\pi$

Џ Article views: 6

Q View related articles $\square$ 
LONDON, EDINBURGH, AND DUBLIN

\title{
PHILOSOPHICAL MAGAZINE
}

\author{
AND \\ JOURNAL OF SCIENCE. \\ [SIXTH SERIES.] \\ JUNE 1908.
}

LXII. Hamilton's Principle and the Five Aberrations of von Seidel. By Lord RAYLeigh, O.M., Pres.R.S.*

I ARGELY owing to the fact that the work of Hamilton, and it may be added of Coddington, remained unknown in Germany and that of v. Seidel in England, it has scarcely been recognized until recently how easily v. Seidel's general theorems relating to optical systems of revolutions may be deduced from Hamilton's principle. The omission has been supplied in an able discussion by Schwarzschild, who expresses Hamilton's function in terms of the variables employed by Seidel, thus arriving at a form to which he gives the name of Seidel's Eikonal $\dagger$. It is not probable that Schwarzschild's investigation can be improved upon when the object is to calculate complete formulæ applicable to specified combinations of lenses; but I have thought that it might be worth while to show how the number and nature of the five constants of aberration can be deduced almost instantaneously from Hamilton's principle, at any rate if employed in a somewhat modified form.

When we speak, as I think we may conveniently do, of five constants of aberration, there are two things which we should remember. The first is that the five constants do not stand upon the same level. By this I mean, not merely that some of them are more important in one instrument and some

* Communicated by the Author.

t The word Eikonal was introduced by Bruns.

Phil. Mag. S. 6. Vol. 15. No. 90. June 1908. 
in another, but rather that the nature of the errors is different. In earlier writings the term aberration was, I think, limited to imperfect focussing of rays which, issuing from one point, converge upon another. Three of the five aberrations are of this character ; but the remaining two relate, not to imperfections of focussing, but to the position of the focus. It is, in truth, something of an accident that, e.g. in photography, we desire to focus distant objects upon a plane. The second thing to which I wish to refer is that, although Seidel did much, four out of the five aberrations were pretty fully discussed by Airy and Coddington before his time. To these authors is due the rule relating to the curvature of images, generally named after Petzval, so far, at any rate, as it refers to combinations of thin lenses.

Some remarks are appended having reference to systems of less highly developed symmetry.

According to Hamilton's original definition of the characteristic function $\mathrm{V}$, it represents the time taken by light to pass from an initial point $\left(x^{\prime}, y^{\prime}, z^{\prime}\right)$ to a final point $(x, y, z)$, and it may be taken to be $\int \mu d s$, where $\mu$ is the refractive index and the integration is along the course of the ray which connects the two points. If the path be varied, the integral is a minimum for the actual ray; and from this it readily follows that

$$
\begin{aligned}
& l=d \mathrm{~V} / d x, \quad m=d \mathrm{~V} / d y, \quad n=d \mathrm{~V} / d z, . \\
& -l^{\prime}=d \mathrm{~V} / d x^{\prime}, \quad-m^{\prime}=d \mathrm{~V} / d y^{\prime}, \quad-n^{\prime}=d \mathrm{~V} / d z^{\prime}, .
\end{aligned}
$$

where $l, m, n, l^{\prime}, m^{\prime}, n^{\prime}$ are the direction-cosines of the ray at the end and beginning of its course, the terminal points being situated in a part of the system where the refractive index is unity.

In his communication to the British Association (B. A. Report, Cambridge 1833, p. 360) Hamilton transforms these equations. As his work is so little known, it may be of interest to quote in full the principal paragraph, with a slight difference of notation :- "When we wish to study the properties of any object-glass, or eye-glass, or other instrument in vacuo, symmetric in all respects, about one axis of revolution, we may take this for the axis of $z$, and we shall have the equations (1), (2), the characteristic function $\mathrm{V}$ being now a function of the five quantities, $x^{2}+y^{2}, x x^{\prime}+y y^{\prime}$, $x^{\prime 2}+y^{\prime 2}, z, z^{\prime}$, involving also, in general, the colour, and having its form determined by the properties of the instrument of revolution. Reciprocally, these properties of the 
instrument are included in the form of the characteristic function $\mathrm{V}$, or in the form of this other connected function,

$$
\mathrm{T}=l x+n y+n z-l^{\prime} x^{\prime}-m^{\prime} y^{\prime}-n^{\prime} z^{\prime}-\mathrm{V}, .
$$

which may be considered as depending on only three independent variables besides the colour; namely, on the inclinations of the final and initial portions of a luminous path to each other and to the axis of the instrument. Algebraically, $\mathrm{T}$ is in general a function of the colour and of the three quantities, $l^{2}+m^{2}, l l^{\prime}+m m^{\prime}, l^{\prime 2}+m^{\prime 2}$; and it may usually (though not in every case) be developed according to ascending powers, positive and integer, of these three latter quantities, which in most applications are small, of the order of the squares of the inclinations. We may therefore in most cases confine ourselves to an approximate expression of the form

$$
\mathrm{T}=\mathrm{T}^{(0)}+\mathrm{T}^{(2)}+\mathrm{T}^{(4)}, \quad . \quad \cdot \quad \cdot \quad .
$$

in which $\mathrm{T}^{(0)}$ is independent of the inclinations ; $\mathrm{T}^{(2)}$ is small of the second order, if those inclinations be small, and is of the form

$$
\mathrm{T}^{(2)}=\mathrm{P}\left(l^{2}+m^{2}\right)+\mathrm{P}_{1}\left(l l^{\prime}+m m^{\prime}\right)+\mathrm{P}^{\prime}\left(l^{\prime 2}+m^{\prime 2}\right) ; .
$$

and $\mathrm{T}^{(4)}$ is small of the fourth order, and is of the form

$$
\begin{aligned}
\mathrm{T}^{(4)}= & \mathrm{Q}\left(l^{2}+m^{2}\right)^{2}+\mathrm{Q}_{1}\left(l^{2}+m^{2}\right)\left(l l^{\prime}+m m^{\prime}\right) \\
& +\mathrm{Q}^{\prime}\left(l^{2}+m^{2}\right)\left(l^{\prime 2}+m^{\prime 2}\right)+\mathrm{Q}_{11}\left(l l^{\prime}+m m^{\prime}\right)^{2} \\
& +\mathrm{Q}_{1}^{\prime}\left(l l^{\prime}+m m^{\prime}\right)\left(l^{\prime 2}+m^{\prime 2}\right)+\mathrm{Q}^{\prime \prime}\left(l^{\prime 2}+m^{\prime 2}\right)^{2} ; .
\end{aligned}
$$

the nine coefficients, $\mathrm{P} \mathrm{P}_{1} \mathrm{P}^{\prime} \mathrm{Q} \mathrm{Q}_{1} \mathrm{Q}^{\prime} \mathrm{Q}_{11} \mathrm{Q}_{1}^{\prime} \mathrm{Q}^{\prime \prime}$, being either constant, or at least only functions of the colour. The optical properties of the instrument, to a great degree of approximation, depend usually on these nine coefficients and on their chromatic variations, because the function $T$ may in most cases be very approximately expressed by them, and because the fundamental equations (1), (2) may rigorously be thus transformed;

$$
\left.\begin{array}{l}
x-\frac{l}{n} z=\frac{d \mathrm{~T}}{d l}, \quad \imath-\frac{m}{n} z=\frac{d \mathrm{~T}}{d m} ; \\
x^{\prime}-\frac{l^{\prime}}{n^{\prime}} z^{\prime}=-\frac{d \mathrm{~T}}{d l^{\prime}}, \quad y-\frac{m^{\prime}}{n^{\prime}} z^{\prime}=-\frac{d \mathrm{~T}}{d m^{\prime}} ;
\end{array}\right\} .
$$

The first three coefficients, $\mathrm{P} \mathrm{P}_{1} \mathrm{P}^{\prime}$, which enter by (5) into the expression of the term $\mathrm{T}^{(2)}$, are those on which the focal lengths, the magnifying powers, and the chromatic aberrations depend: the spherical aberrations, whether for $2 \mathrm{Z} 2$ 
direct or inclined rays, from a near or distant object, at either side of the instrument (but not too far from the axis), depend on the six other coefficients, $Q Q_{1} Q^{\prime} Q_{11} Q_{1}^{\prime} Q^{\prime \prime}$, in the expression of the term $\mathrm{T}^{(4)}$. Here, then, we have already a new and remarkable property of object-glasses, and eye-glasses, and other optical instruments of revolution; namely, that all the circumstances of their spherical aberrations, however varied by distance and inclination, depend (usually) on the values of SIX RADICAL CONSTANTS OF ABERRATION, and may be deduced from these six numbers by uniform and general processes. And as, by employing general symbols to denote the constant coefficients or elements of an elliptic orbit, it is possible to deduce results extending to all such orbits, which can afterwards be particularized for each ; so, by employing general symbols for the six constants of aberration, suggested by the foregoing theory, it is possible to deduce general results respecting the aberrational properties of optical instruments of revolution, and to combine these results afterwards with the peculiarities of each particular instrument by substituting the numerical values of its own particular constants."

Equations (7) are easily deduced. So far as it depends upon the unaccented letters, the total variation of $\mathrm{T}$ is

$$
\begin{aligned}
d \mathrm{~T}=l d x+m d y+n d z & +x d l+y d m+z d n \\
& -\frac{d \mathrm{~V}}{d x} d x-\frac{d \mathrm{~V}}{d y} d y-\frac{d \mathrm{~V}}{d z} d z,
\end{aligned}
$$

or regard being paid to (1),

in which

$$
d \mathrm{~T}=x d l+y d m+\approx d n,
$$

so that

$$
l d l+n d m+n d n=0,
$$

$$
\frac{d \mathrm{~T}}{d l}=a^{\prime}-\frac{l z}{n}, \quad \frac{d \mathrm{~T}}{d m}=y-\frac{m z}{n} ;
$$

and in like manner by varying the accented letters the second pair of equations ( 7 ) follows.

If we agree to neglect the cubes of the inclinations, we may identify $n, n^{\prime}$ with unity, and (7) becomes

$$
\begin{array}{ll}
x=(z+2 \mathrm{P}) l+\mathrm{P}_{1} l^{\prime}, & y=(z+2 \mathrm{P}) m+\mathrm{P}_{1} m^{\prime}, \\
x^{\prime}=-\mathrm{P}_{1} l+\left(z^{\prime}-2 \mathrm{P}^{\prime}\right) l^{\prime}, & y^{\prime}=-\mathrm{P}_{1} m+\left(z^{\prime}-2 \mathrm{P}^{\prime}\right) m^{\prime},
\end{array}
$$

determining $x, x^{\prime}$ in terms of $z, z^{\prime}, l, l^{\prime}$ supposed known, or conversely $l, l^{\prime}$ in terms of $z, z^{\prime}, x, t^{\prime}$ supposed known. The 
case of special interest is that in which $x, y, z$ and $x^{\prime}, y^{\prime}, z^{\prime}$ are conjugate points, i.e. images of one another in the optical system. The ratio $x: x^{\prime}$ must then be independent of the special values ascribed to $l, l^{\prime}$. In order that this may be possible, i.e. in order that $z, z^{\prime}$ may be conjugate planes, the condition is

and then

$$
(z+2 \mathrm{P})\left(z^{\prime}-2 \mathrm{P}^{\prime}\right)+\mathrm{P}_{1}^{2}=0, \ldots . .
$$

$$
\frac{x}{x^{\prime}}=\frac{y}{y^{\prime}}=-\frac{z+2 \mathrm{P}}{\mathrm{P}_{1}}=\frac{\mathrm{P}_{1}}{z^{\prime}-2 \mathrm{P}^{\prime}}, \quad . \quad . \quad \text {. }
$$

giving the magnification.

Equations (8), (9) express the theory of a symmetrical instrument to a first approximation. In order to proceed further we should have not only to include the terms in (7) arising from $\mathrm{T}^{(4)}$, but also to introduce a closer approximation for $n$. Thus even though $\mathrm{T}^{(4)}=0$, we should have additional terms in the expressions for $x, x^{\prime}$ equal respectively to

$$
\frac{1}{2} l z\left(l^{2}+m^{2}\right) \text { and } \frac{1}{2} l^{\prime} z^{\prime}\left(l^{\prime 2}+m^{\prime 2}\right) \text {. }
$$

If the object is merely to express the aberrations for a single pair of conjugate planes, we may attain it more simply by a modification of Hamilton's process.

Supposing that the conjugate planes are $z=0, z^{\prime}=0$, we have $V$ a function of the coordinates of the initial point $x^{\prime}, y^{\prime}$, and of the final point $x, y$. And if as before $l, m, n, l^{\prime}, m^{\prime}, n^{\prime}$ are the direction-cosines of the terminal portions of the ray, we still have

$$
\begin{array}{ll}
l=d \mathrm{~V} / d x, & m=d \mathrm{~V} / d y, \ldots \\
l^{\prime}=-d \mathrm{~V} / d x^{\prime}, & m^{\prime}=-d \mathrm{~V} / d y^{\prime} .
\end{array}
$$

But now instead of transforming to a function of $l, m$, $l^{\prime}, m^{\prime}$, from which $x^{\prime}, y^{\prime}, x, y$ are eliminated, we retain $x^{\prime}, y^{\prime}$ as independent variables, eliminating only $x, y$, the coordinates of the final or image point*. For this purpose we assume

$$
\mathrm{U}=l x+m y-\mathrm{V} . \quad . \quad . \quad . \quad .
$$

The total variation of $\mathrm{U}$ is given by

$$
\begin{aligned}
d \mathrm{U}=x d l & +l d x+y d m+m d y \\
& -\frac{d \mathrm{~V}}{d x} d x-\frac{d \mathrm{~V}}{d y} d y-\frac{d \mathrm{~V}}{d x^{\prime}} d x^{\prime}-\frac{d \mathrm{~V}}{d y^{\prime}} d y^{\prime},
\end{aligned}
$$

or with regard to $(10),(11)$

$$
d \mathrm{U}=x d l+y d m+l^{\prime} d x^{\prime}+m^{\prime} d y^{\prime}, \quad . \quad .
$$

* Compare Routh’s ‘ Elementary Rigid Dynamics,' § 418. 
from which it appears that $\mathrm{U}$ is in reality a function of $x^{\prime}, y^{\prime}$, $l, m$. As equivalent to (13), we have

$$
\begin{array}{llll}
x=d \mathrm{U} / d l, & y=d \mathrm{U} / d m, & . & . \\
l^{\prime}=d \mathrm{U} / d x^{\prime}, & m^{\prime}=d \mathrm{U} / d y^{\prime} . & . & .
\end{array}
$$

So far $U$ appears as a function of the four variables $x^{\prime}, y^{\prime}$, $l, m$; but from its nature, as dependent upon $l x+m y$ and $V$, and from the axial symmetry, it must be in fact a function of the three variables

$$
x^{\prime 2}+y^{\prime 2}, \quad l^{2}+m^{2}, \quad \text { and } \quad l x^{\prime}+m y^{\prime},
$$

the latter determining the angle between the directions of $x^{\prime}, y^{\prime}$ and $l, m$. When these quantities are small, we may take

$$
\mathrm{U}=\mathrm{U}^{(0)}+\mathrm{U}^{(2)}+\mathrm{U}^{(4)}+\ldots, \ldots .
$$

where $\mathrm{U}^{(0)}$ is constant and

$$
\mathrm{U}^{(2)}=\frac{1}{2} \mathrm{~L}\left(l^{2}+m^{2}\right)+\mathrm{M}\left(x^{\prime} l+y^{\prime} m\right)+\frac{1}{2} \mathrm{~N}\left(x^{\prime 2}+y^{\prime 2}\right), .
$$

$\mathrm{L}, \mathrm{M}, \mathrm{N}$ being constants. If we stop at $\mathrm{U}^{(2)}$, equations (14) give

$$
x=\mathrm{L} l+\mathrm{M} x^{\prime}, \quad y=\mathrm{I} m+\mathrm{M} y^{\prime}, \quad .
$$

determining $x, y$ as functions of $x^{\prime}, y^{\prime}, l, m$. We have next to introduce the supposition that $x, y$ is conjugate to $x^{\prime}, y^{\prime}$. Hence $\mathrm{L}=0$, for to this approximation $x, y$ must be determined by $x^{\prime}, y^{\prime}$ independently of $l, m$. Accordingly,

$$
x=\mathrm{M} x^{\prime}, \quad y=\mathrm{M} y^{\prime} . \quad \text {. . . . }
$$

We are now prepared to proceed to the next approximation. In order to correspond, as far as may be, with the notation of Seidel * we will write

$$
\begin{aligned}
\mathrm{U}^{(4)}= & \frac{1}{4} \mathrm{~A}\left(l^{2}+m^{2}\right)^{2}+\mathrm{B}\left(l^{2}+m^{2}\right)\left(l x^{\prime}+m y^{\prime}\right) \\
& +\frac{1}{2}(\mathrm{C}-\mathrm{D})\left(l x^{\prime}+m y^{\prime}\right)^{2}+\frac{1}{2} \mathrm{D}\left(l^{2}+m^{2}\right)\left(x^{\prime 2}+y^{\prime 2}\right) \\
& +\mathrm{E}\left(l x^{\prime}+m y^{\prime}\right)\left(x^{\prime 2}+y^{\prime 2}\right)+\mathrm{F}\left(x^{\prime 2}+y^{\prime 2}\right)^{2}, . .
\end{aligned}
$$

which is the most general admissible function of the fourth degree.

From (20) we obtain by use of (14) the additional terms in $x$ and $y$ dependent on $U^{(4)}$. No generality is lost if at this stage we suppose, for the sake of brevity, $y^{\prime}=0$. Accordingly,

$$
\begin{aligned}
& x=\mathrm{A} l\left(l^{2}+m m^{2}\right)+\mathrm{B} x^{\prime}\left(3 l^{2}+n x^{2}\right)+\mathrm{C} x^{\prime 2} l+\mathrm{E} x^{\prime 3}, . \\
& y=\mathrm{A} m\left(l^{2}+m^{2}\right)+2 \mathrm{~B} \cdot c^{\prime} l m+\mathrm{D} x^{\prime 2} m . \quad . \quad . \quad .
\end{aligned}
$$

* Finsterwalder, München. Sitz. Ber. xxvii. p. 408 (1897). 
In order to complete the value of $x$ we must add the expressions in (19) and (21).

Since $F$ disappears from the values of $x$ and $y$, we see that there are five effective constants of aberration of this order, as specified by Seidel. The evanescence of $\mathrm{A}$ is the Eulerian condition for the absence of spherical aberration in the narrower sense, i.e. as affecting the definition of points lying upon the axis $\left(x^{\prime}=0\right)$. If the Eulerian condition be satisfied, $B=0$ is identical with what Seidel calls the Fraunhofer condition*. The theoretical investigation of this kind of aberration was one of Seidel's most important contributions to the subject, inasmuch as neither Airy nor Coddington appears to have contemplated it. The conditions $\mathrm{A}=0, \mathrm{~B}=0$ are those which it is most important to satisfy in the case of the astronomical telescope.

To this order of approximation $B=0$ is identical with the more general sine condition of Abbe, which prescribes that, in order to the good definition of points just off the axis, a certain relation must be satisfied between the terminal inclinations of the rays forming the image of a point situated on the axis. The connexion follows very simply from the equations already found. By (15), (16), (17), (20), with $m=0$,

$$
l^{\prime}=\mathrm{M} l+\mathrm{B} l^{3}+\text { terms vanishing with } x^{\prime}, y^{\prime} \text {; }
$$

so that for the conjugate points situated upon the axis

$$
l^{\prime}=\mathrm{M} l+\mathrm{B} l^{3} .
$$

The condition $\mathrm{B}=0$ is thas equivalent to a constant value of the ratio $l^{\prime} / l$, that is the ratio of the sines of the terminal inclinations of a ray with the axis. And this is altogether independent of the value of $\mathrm{A}$.

On the supposition that the two first conditions $A=0$, $\mathrm{B}=0$ are satisfied, we have next to consider the significance of the terms multiplied by $\mathrm{C}$ and $\mathrm{D}$. Since

$$
d x / d l=\mathrm{C} x^{\prime 2}, \quad d y / d m=\mathrm{D} x^{\prime 2},
$$

we see that $\mathrm{C}$ and $\mathrm{D}$ represent departures of the primary and secondary foci from the proper plane. In fact if $1 / \rho_{1}, 1 / \rho_{2}$

* If $A$ be not equal to zero, it can be shown that the best focussing of points just off the axis requires that

$$
\mathrm{A} l_{0}+\mathrm{B} x^{\prime}=0,
$$

where $l_{0}$ is the value of $l$ for the principal ray. For example, if the optical system reduces to a combination of thin lenses close together, $l_{0}=x / f$, where $f$ is the distance of the lenses from the image plans. Since by (19), $x=\mathrm{M} \cdot x^{\prime}$, the condition may be written

$$
\mathrm{AM}+\mathrm{B} f=0 \text {. }
$$


be the curvatures of the images, as formed by rays in the two planes,

$$
1 / \rho_{1}=2 \mathrm{C}, \quad 1 / \rho_{2}=2 \mathrm{D} . \quad . \quad .
$$

The condition of astigmatism is then

$$
\mathrm{C}=\mathrm{D} \text {; . . . . . . }
$$

but unless both constants vanish the image is curved.

Finally the term containing $\mathrm{E}$ represents distortion.

If we impose no restriction upon the values of the constants of aberration, we have in general from (21), (22)

$$
\begin{aligned}
d x / d l & =\mathrm{A}\left(3 l^{2}+m^{2}\right)+6 \mathrm{~B} x^{\prime} l+\mathrm{C} x^{\prime 2}, \\
d y / d m & =\mathrm{A}\left(l^{2}+3 m^{2}\right)+2 \mathrm{~B} x^{\prime} l+\mathrm{D} x^{\prime 2} .
\end{aligned}
$$

These equations may be applied to find the curvatures of the image as formed by rays infinitely close to given rays, as for example when the aperture is limited by a narrow stop placed centrally on the axis, but otherwise arbitrarily. The principal ray is then characterized by the condition $m=0$, and we have

$$
\begin{aligned}
& d x / d l=3 \mathrm{~A} l^{2}+6 \mathrm{~B} x^{\prime} l+\mathrm{C} x^{\prime 2}=3 \mathrm{H}+\mathrm{K}, . . \\
& d y / d m=\mathrm{A} l^{2}+2 \mathrm{~B} x^{\prime} l+\mathrm{D} x^{\prime 2}=\mathrm{H}+\mathrm{K}, . .
\end{aligned}
$$

equations which determine the curvatures of the images as formed by rays in the neighbourhood of the given one, and deviating from it in the primary and secondary planes respectively.

$$
\begin{aligned}
& \text { According to }(26),(27), \\
& \qquad \begin{array}{l}
2 \mathrm{H}=2 \mathrm{~A} l^{2}+4 \mathrm{~B} x^{\prime} l+(\mathrm{C}-\mathrm{D}) x^{\prime 2}, . . \\
2 \mathrm{~K}=(3 \mathrm{D}-\mathrm{C}) x^{\prime 2} \quad . \quad . \quad . \quad . \quad .
\end{array}
\end{aligned}
$$

The requirement of flatness in both images is thus satisfied if $\mathrm{H}=0, \mathrm{~K}=0$. The former is the condition of astigmatism, and it involves the ratio of $x^{\prime}: l$, which is dependent upon the position of the stop ; but the latter does not depend on this ratio. It corresponds to the condition formulated by Coddington and later by Petzval. From (28), (29) we may of course fall back upon the conditions already laid down for the case where $A=0, B=0$.

The further pursuit of this subject requires a more particular examination of what occurs when light is refracted at spherical surfaces. Reference may be made to Schwarzschild *, who uses Hamilton's methods as applied to a special form of the characteristic function designated as Seidel's Eikonal. A concise derivation of the Coddington-Petzval

* Göttingen $A b h$. iv. 1905. 
condition by elementary methods will be found in Whittaker's tract *.

Before leaving systems symmetrical about, an axis to which all the rays are inclined at small angles, we may remark that, as $I^{(4)}$ contains 6 constants, in like manner $U^{(6)}$ contains 10 constants $\dagger$ and $\mathrm{U}^{(8)} 15$ constants, of which in each case one is ineffective.

The angle embraced by some modern photographic lenses is so extensive that a theory which treats the inclinations as small can be but a rough guide. It remains true, of course, that an absolutely flat field requires the fulfilment of the Coddington-Petzval condition; but in practice some compromise has to be allowed, and this involves a sacrifice of complete flatness at the centre of the image. It will be best to fulfil the conditions $d x / d l=0, d y / d m=0$, or, what are equivalent,

$$
d^{2} \mathrm{U} / d l^{2}=0, \quad d^{2} \mathrm{U} / d m^{2}=0,
$$

not when $l$ is very small but when it attains some finite specified value. If we suppose $y^{\prime}=0, \mathrm{U}$ is a function of $x^{\prime 2}, l^{2}+m^{2}$, and $l x^{\prime}$, or say of $u, v, w$. Hence

$$
\begin{aligned}
& \frac{d^{2} \mathrm{U}}{d l^{2}}=4 l^{2} \frac{d^{2} \mathrm{U}}{d v^{2}}+2 \frac{d \mathrm{U}}{d v}+x^{\prime 2} \frac{d^{2} \mathrm{U}}{d c^{2}}+4 x^{\prime} l \frac{d^{2} \mathrm{U}}{d \bar{w} d v}, \\
& \frac{d^{2} \mathrm{U}}{d m^{2}}=4 m^{2} \frac{d^{2} \mathrm{U}}{d v^{2}}+2 \frac{d \mathrm{U}}{d v} .
\end{aligned}
$$

After the differentiations are performed, we are to make $m=0$; so that the two conditions of astigmatism and focus upon the plane, analogous to (28), (29), are

$$
\frac{d \mathrm{U}}{d v}=0, \quad 4 l^{2} \frac{d^{2} \mathrm{U}}{d v^{2}}+x^{2} \frac{d^{2} \mathrm{U}}{d w^{2}}+4 x^{\prime} l \frac{d^{2} \mathrm{U}}{d v d w}=0,
$$

in which $v$ is to be made equal to $l^{2}$. But it is doubtful whether such equations could be of service.

Let us now suppose that the system is indeed symmetrical with respect to the two perpendicular planes of $x$ and $y$, but not necessarily so round the axis of $z$. In the expression for $\mathrm{U}$ no terms can occur which would be altered by a simultaneous reversal of $x^{\prime}$ and $l$, or of $y^{\prime}$ and $m$. For $U^{(2)}$ we have

$$
\begin{aligned}
\mathrm{U}^{(2)}=\alpha l^{2}+\beta m^{2}+\gamma x^{\prime} l+\delta y^{\prime} m \\
\\
+ \text { terms independent of } l \text { and } m .
\end{aligned}
$$

* 'Theory of Optical Instruments,' Cambridge, 1907. The optical invariants, introduced by Abbe, are there employed.

$\dagger$ Schwarzschild, loc. cit. 
686 Hamilton's Principle and the Five Aberrations of $v$. Seidel.

Hence, by (14),

$$
x=2 \alpha l+\gamma x^{\prime}, \quad y=2 \beta m+\delta y^{\prime} .
$$

If $x, y$ is conjugate to $x^{\prime}, y^{\prime}$, we must have

so that

$$
\alpha=0, \quad \beta=0 ;
$$

$$
x=\gamma x^{\prime}, \quad y=\delta y^{\prime} . \quad . \quad-. .
$$

These are the equations of the first approximation, and they indicate that the magnification need not be the same in the two directions.

There are no terms in $\mathrm{U}^{(3)}$. As regards $\mathrm{U}^{(4)}$, we have

$$
\begin{aligned}
\mathrm{U}^{(4)} & =\mathrm{A} l^{4}+\mathrm{B} l^{2} m^{2}+\mathrm{C} m^{4} \\
& +\mathrm{D} x^{\prime} l^{3}+\mathrm{E} x^{\prime} l m^{2}+\mathrm{F} y^{\prime} m^{3}+\mathrm{G} y^{\prime} l^{2} m \\
& +\mathrm{H} x^{\prime 2} l^{2}+\mathrm{I} x^{\prime 2} m^{2}+\mathrm{J} x^{\prime} y^{\prime} l m+\mathrm{K} y^{\prime 2} l^{2}+\mathrm{L} y^{\prime 2} m^{2} \\
& +\mathrm{M} x^{\prime 3} l+\mathrm{N} x^{\prime 2} y^{\prime} m+\mathrm{O} x^{\prime} y^{\prime 2} l+\mathrm{P} y^{\prime 3} m \\
& + \text { terms independent of } l \text { and } m . . . . . .
\end{aligned}
$$

In (31) there are 16 effective constants as compared with 5 in the case where the symmetry round the axis is complete; so that such symmetry implies 11 relations among the constants of (31). For example, in the terms of the first line representing Eulerian aberration, axial symmetry requires that

$$
\mathrm{C}=\frac{1}{2} \mathrm{~B}=\mathrm{A} \text {. }
$$

We will next suppose that the only symmetry to be imposed is that with respect to the primary plane $y=0$; so that $U$ is unchanged it the signs of $y^{\prime}$ and $m$ are both reversed. $\mathrm{U}^{(2)}$ is of the same form as in the case of double symmetry, and

$$
x=2 \alpha l+\gamma x^{\prime}, \quad y=2 \beta m+\delta y^{\prime} .
$$

If $x, y$ is the image of $x^{\prime}, y^{\prime}$, formed by rays in both planes, $\alpha=0, \beta=0$, as before. 'But it may happen, e.g. in the spectroscope, that there is astigmatism even in the first approximation. If the points are images of one another as constituted by rays in the primary plane, $\alpha=0$, but $\beta$ is left arbitrary.

The next term in $U$ may be denoted by $U^{(3)}$. If no conditions of symmetry were imposed, $\mathrm{U}^{(3)}$ would include 16 effective terms, $i$. $e$. terms contributing to $x, y$; but the 
symmetry with respect to $y=0$ excludes 8 of these. We may write

and, by (14),

$$
\begin{aligned}
\mathrm{U}^{(3)} & =a l^{3}+b l m^{2} \\
& +c x^{\prime} l^{2}+d x^{\prime} m^{2}+e y y^{\prime} l m \\
& +f x^{\prime 2} l+g x^{\prime} y^{\prime} m+h y^{\prime 2} l, \quad . \quad . \quad . .
\end{aligned}
$$

$$
x=3 a l^{2}+b m^{2}+2 c x^{\prime} l+e y^{\prime} m+f x^{\prime 2}+h y^{\prime 2} .
$$

If the rays all proceed from the point $x^{\prime}=0, y^{\prime}=0$, the conditions for a well-formed primary focal line are

$$
a=0, \quad b=0, \text {. . . . . }
$$

of which the first expresses that there is no aberration of this order for rays in the primary plane, $i . e$., that the focal line is thin, while the second is the condition that the focal line is straight*.

But if, while $x^{\prime}=0, y^{\prime}$ be left arbitrary, so that the source of light is linear, the evanescence of (34) requires, in addition to $(35)$, that

$$
e=0, \quad h=0 . \quad \text {. . . . . }
$$

Terling Place, Witham, April 20, 1908.

\section{The Problem of a Spherical Gaseous Nebula.} By the late Lord KELvin $\dagger$.

THIs paper was begun about the close of 1906 , in order to fulfil a promise given at the end of the paper "On the Convective Equilibrium of a Gas under its own Gravitation only," published in the Philosophical Magazine, 1887; and part of it was communicated by Lord Kelvin to the Royal Society of Edinburgh at its meeting on the 21 st January 1907 . Since then, how ever, important additions have been made to it, and the subject has been dealt with more fully than was originally intended. Unfortunately the manuscript was left incomplete at Lord Kelvin's death. It ended with $\S 35$.

However, from information which I received from Lord Kelvin while carrying out the earlier work connected with the paper, I have been able to write the sections from $\$ 36$ to the end. These complete all that Lord Kelvin desired to include in this communication;

* Compare Phil. Mag. vol. viii. p. 481, 1879; Scientific Papers, i. p. 440.

† Communicated by Dr. J. T. Bottomley, F.R.S. 